\title{
Giornale italiano delle malattie veneree e della pelle. Heft 5. 1910.
} pag. 645 .

Martinotti, L. Die Plasmazellen. Heft 4. pag. $ّ 22$ und Heft 5 .

Sehr ausführliche Arbeit, die in einer Reihe von Kapiteln eine Zusammenfassung alles Wissenswerten über die Plasmazellen enthält. (Technik der Demonstration der Plasmazellen, ihre Morphologie, die Varietäten und Nomenklatur derselben, die degenerativen Alterationen, das Vorkommen der Plasmazellen in den normalen und pathologischen Geweben, im Blut, ihre Differentialdiagnose, ferner die Plasmazellen bei experimentellen Untersuchungen, die alten und modernen Theorien ihres Ursprungs, ihre Bedeutung und ihr Geschick.)

Nach der Beschreibung aller von ihm selbst und von anderen Autoren vorgenommenen Untersuchungen kommt Martinotti zu dem Schlub, daß die Plasmazellen ein morphologisch gut definiertes und in den normalen und pathologischen Geweben äußerst verbreitetes Element darstellen. Sie können in der Norm die Zellen des retikulären (besonders des lymphoiden) und des konnektivalen Gewebes begleiten, denen sie in bezug auf ihren Ursprung innig verbunden sind. Sie nehmen fast konstant Teil an den Zellanhäufungen der Entzändung, vor allem an den chronischen.

Sie zeigen sich unter verschiedenen Typen oder Varietäten, nämlich dem lymphozytären Typus (oder Plasmatozyten), der bei weitem häufiger vorkommt als die anderen, ferner dem lymphoblastischen (großem lymphozytären) Typus, der weniger verbreitet ist als der. vorkergebende, und schließlich unter der Form vou kleinen Plasmazellen und splenoiden oder splenoblastischen Plasmazellen (große mononukleäre), die außerordentlich viel seltener sind.

In bezug aut die Genese ist zu bemerken, daß im Laufe der ontogenetischen Entwicklung sich vom primären Mesenchym zwei Gewebe differenziert haben, nämlich das retikuläre; das wesentlich dem Zwecke dient, die Elemente des Lymph- und Blutgefäßsystems zu produzieren, und das konnektivale Gewebe im eigentlichen Sinne, das vor allem zu der Funktion bestimmt ist, den anderen Geweben, den Gefäßen und Organen als Stütze zu dienen.

In dem ersten Gewebe bewahrt die ganze Serie der fixen Elemente, die von den.jungen, die Kapillarwandungen bildenden Endothelzellen zu den der Adventitia der Gefäße aufliegenden Zellen, zu den großen Zellen des Retikulums geht, auch beim Erwachsenen die. Fähigkeit entweder durch Multiplikation mit Erwerbung neuer zytogenetischer. Charaktere (metaplastische Proliferation) oder durch individuelle differentielle Transformation (heteroplastische Differenzierung) bewegliche Elemente des lymphoiden Parenchyms hervorzubringen.

Bei dem konnektivalen Gewebe dagegen haben beim Erwachsenen nur die jüngsten Zellformen (junge Fibroblasten oder Plasmatozyten, 
oder Adventitiazellen), die meist um die Gefäße herum lokalisiert sind, eine solche Fähigkeit bewahrt, während die mehr entwickelten Formen (erwachsene Fibroblasten) schon differenzierte Elemente sind, wesentlich zur Produktion des Kollagens bestimmt. Und es ist poch nicht bewiesen, daß sie unter bestimmten Bedingungen zum embryonalen $Z$ ustand zurückkehren können, indem sie eine erhöhte Differenzierungskraft erlangen, d. h. wenigstens ohne zuerst eine Generation junger Elemente durch direkte oder indirekte Multiplikation hervorgebracht zu haben.

Wenn ein entzündlicher Zustand eintritt, so findet eine Einwanderung mono- und polynukleärer Elemente (lymphato-myelogenen Ursprungs) durch die Gefäße hindurch statt, und eine lokale Reaktion vonseiten der jungen konnektivalen Elemente, welche einen Komplex von runden, den eingewanderten vollkommen identischen Elementen ergeben. (Ausgenommen ind vielleicht die Mastzellen und die eosinophilen Zellen, die hauptsächlich mononukleäre Elemente zu sein scheinen, im Gegensatz zu den homologen polynukleären Elementen des Blutes. Es ist jedoch nicht auszuschließen, daß auch sie in ihrer weiteren Entwicklung die polynukleäre Form annehmen und sich dann n:cht mehr von den hämatogenen Elementen unterscheiden lassen). Die erwachsenen Fibroblasten dagegen ergeben die großen hyperbasophilen Fibroblasten der entzündeten Gewebe.

Wir befinden uns so gegenüber Elementen hämatogenen und histiogenen Ursprungs, welche morphologisch gleich sind, und die getrennt zu halten kein Grund besteht, um so mehr als sie außer der strukturellen Gleichbeit auch innige genetische Beziehungen darbieten, insofern als sie das Produkt von Zellen desselben Ursprungs sind.

Von diesem Komplex runder histiogener und lymphato myelogener Zellen rühren die Plasmazellen her, welche entweder direkt von den jungen Zellen des retikulären und konnektivalen Gewebes stammen oder zuerst das Stadium der mononukleären Elemente passieren.

Wenn man die Entwicklung all dieser Elemente näher verfolgen will, so kann man folgendes feststellen:

1. Durch einfaches zytogenetisches (homoplastisches) Wachstum mit Akzentuierung der schon besessenen Charaktere entwickeln sich vier Serien von Elementen: a) aus den Endothelzellen die Zellen des Retikulums; $b$ ) aus den jungen Fibroblasten (Adventitiazellen) die erwachsenen Fibroblasten; c) aus den Lymphozyten die Lymphoblasten und aus diesen die Splenozyten; $d$ ) aus den lymphozytären Plasmazcilen die lymphoblastischen, aus diesen die splenoblastischen.

2. Durch differentielle Entwicklung mit Erwerbung neuer zytogenetischer Charaktere (metaplastische oder heteroplastische Differenzierung) können aus den jungen Zellen des Retikulums und des konnektivalen Gewebes Lymphozyter und typische Plasmazellen entstehen; aus den erwachsenen Zellen des Retikulums können bewegliche Elemente (mononukleäre) hervorgehen, während dies für die großen, erwachsenen Fibroblasten des konnektivalen Gewebes noch nicht bewiesen ist. 
In analoger Art kann von der ganzen Serie der mononukleären Elemente die Serie der Plasmazellen abstammen, nämlich von den kleinen Lymphozyten mit unregelmäßigem Kern die kleinen Plasmazellen, von den etwas größeren als den vorhergehenden mit regelmäßigem Kern die Plasmatozyten, von den Lymphoblasten die lymphoblastischen Formen und vor den Splenozyten die splenoblastischen.

Bejm Aufhören der Entzündung geht der größte Teil der runden Zellen (die Plasmazellen einbegriffen) zuräck oder degeneriert und verschwindet.

Die Funktion und Bedeutung der Plasmazellen sind innig gebunden an die der Lymphozyten, von denen sie einen hypertrophischen Zustand. in bezug auf Struktur und Funktion darstellen.

Wegen aller weiteren Darlegungen der Arbeit, der ein ausführliches Literaturverzeichnis und Abbildungeu beigegeben sind, muß anf das Original verwiesen werden.

Stanziale, R. Inokulationen leprösen Materials in die vordere Kammer von Kaninchen. p. 702.

Nach einleitenden Bemerkungen über die von vielen Autoren vorgenommenen Übertragungsversuche der Lepra auf Tiere beschreibt Stanziale ausführlich die Technik und die Ergebnisse der von ihm. selbst angestellten Experimente.

Bei der Inokulation leprösen Materials in die vordere Kammer. waren die. Effekte verschieden, je nachdem die Inokulation mit solidem (Stückchen von Lepraknoten) oder flüssigem Material (Saft von Lepraknoten, der mit Saugglocke aspiriert worden war, oder Suspension von Stückchen eines Lepraknoten in physiol. Lösung) gemacht worden war. (In allen Fällen war das inokulierte Produkt sehr reich an Leprabazillen.)

In der Tat hat die Inokulation von flüssigem Material konstant negatives Resultat bei den einzelnen Untersuchungen ergeben, nicht nur in der vorderen Kammer, sondern auch im subkonjunktivalen Gewebe, durch den Limbus und die Kornea hindurch. Bei allen Tieren, denen flüssiges Material inokuliert wurde, war es schon nach wenigen Tagen bei noch so zahlreichen Untersuchungen niemals möglich, säurefeste bazilläre Formen nachzuweisen.

Die Inokulation von Stückchen leprösen Gewebes in die vordere Kammer führte dagegen stets zur Entwicklung anatomisch-pathologischer Läsionen, dif durch granulomatöse Neoformationen charakterisiert waren. Und die Untersuchung auf Leprabazillen war in diesen Fällen immer positiv. In irgend einer Periode nach der Inokulation waren stets in großer Zahl die verschiedenen Formen (granuläre, bazilläre, in Haufen angeordnete, treie und endozelluläre) des Lepraerregers nachzuweisen.

Die Inokulation leprösen Gewebes war also für A. die Bedingung sine qua non zur Erreichung eines positiven Übertragungsresultates.

In bezug auf die Übertragung der Lepra von Kaninchen auf Kaninchen kann A. noch kein Urteii abgeben (die Frage wird von ihm noch studiert); nur so viel kann er sagen, daß auch in diesem Falle die In- 
okulation flüssigen Materials (wie schon bei den Inokulationsversuchen rom Menschen aufs Kaninchen) negatives Resultat ergibt.

Bei der Inokulation von menschlichen Lepromstückchen in die vordere Kammer des Kaninchens traten zuerst in allen Fällen leichte entzündliche Erscheinungen auf (Imbibition des transplantierten Gewebes, leichte reaktive Exsudation).

Dieser ersten Periode des Turgors folgte konstant zunächst eine Phase der Verkleinerung und dann ein ziemlich lange dauernder stationärer Zustand, der aufgefaßt werden könnte im Sinne einer Anpassung an dem neuen Organismus oder besser im Sinne einer wirklichen Inkubation, die nötig ist, damit der Leprabazillus im Auge spezitische Alterationen hervorbringen kann. Es folgt eine Phase von Wachstum in toto des inokulierten leprösen Gewebes und das Auftreten punktförmiger, makroskopischer Knötchen auf dem inokulierten Material oder in seiner Nachbarschaft. Außer der Anwesenheit von Läsionen mit evident granulomatösem Typus, die sich in den Geweben des Auges zeigten, welche mit dem inokulierten Stück Kontinuitäts- oder Kontiguitätsbeziehungen haben, d. h. in den hinteren Schichten der Kornea und durch die ganze Dicke der Iris hindurch, mub besonders bervorgehoben werden, dab sich bei einem Kaninchen innerhalb der Kornea in einiger Distanz von dem inokulierten Stückchen zirkumskripte granulomatöse Knötchen entwickelten, die charakterisiert waren durch eine periphere Zone kleinzelliger Infiltration und dnrch einen zentralen Bezirk, der hauptsächlich aus epitheloiden Zellen mit lymphoiden Elementen und einigen Riesenzellen bestand.

Bemerkenswerter waren noch die Läsionen bei einem anderen Kaninchen, in dem die Entwicklung granulomatöser Herde sowohl im Irisgewebe statthatte, das in direktem Kontakt mit dem in die vordere Kammer inokulierten Stückchen war, als auch innerhalb der Kornea, entfernt sowohl von der Penetrationsstelle der Lanzette beim operativen Eingriff als auch von der vorderen Kammer selbst. In diesem Falle waren tiefgehende nekrotische Alterationen vorhanden, entsprechend dem zentralen Teile des Granuloms, mit wahren Sequestern nekrotisierten Korneagewebes. Diese nekrotische Phase fehlte fast vollkommen bei den granulomatösen Herden der anderen Kaninchen, weil bei diesen das Auge nach kurzer Zeit, offenbar in der Anfangsphase der Entwicklung dieser Alterationen enukleiert wurde.

Der bakterioskopische Befund war bei allen Kaninchen bei wiederholten Untersuchungen immer positiv in bezug auf das Vorhandensein von extra- und endozellulären Bazillen. Und die Zahl der in den Granulomen gefundenen Bazillen war stets sehr bedentend und in einigen Fällen derart, daß die Multiplikation der inokulierten Keime nicht in Zweifel gezogen werden konnte. Auch in den die neugebildeten Granulome umgebenden Geweben und an anderen Stellen, wo sich noch keine sichtbaren Alterationen fanden, hat $A$. die Anwesenheit zahlreicher in Haufen angeordneter Bazillen feststelien können. 
Man muß annehmen, daß all die makroskopischen und histologischen Manifestationen absolut und ausschließlich in Beziehung stehen zur Anwesenheit des Leprabazillus, der die wahre Ursache der angetroffenen Läsionen bildet.

Die näheren Details der mit vielen anschaulichen Abbildungen ausgestatteten Arbeit müssen im Original nachgesehen werden.

Dalla Favera, G. B. Über zwei Beobachtungen von Lichen nitidus (Pinkus). p. 738.

Dalla Favera gibt eine klinische und histologische Beschreibung zweier Fälle der durch Pinkus unter dem-Namen Lichen nitidus bekannt gewordenen Dermatose. Wie die Affektion von den Follikeln der Penisund Skrotumhaut, von flachen verrucae juveniles, von Molluscum con. tagiosum und besonders von Lichen ruber planus Wilson, der ein ähnliches klinisches Bild liefern kann, zu unterscheiden ist, wird von $A$. besprochen.

Das histologische Substrat des Lichen nitidus hat Ähnlichkeit mit Lichen. scrophulosorum und Syphilis miliopapulosa. Aber bei letzteren Affektionen ist die Lokalisation des Infiltrates um die Follikel die Regel, während sie beim Lichen nitidus eine Ausnahme bildet. Bei jenen sind außerdem die Zeichen der Entzündung bedeutend mehr ausgesprochen als bei diesem.

In bezug auf die histologischen Differenzen zwischen Lichen nit. und L. planus kann A. die Befunde von Pinkus und Arndt bestätigen. Das Infiltrat bei Lichen nit. ist kompakt, scharf begrenzt gegen das umgebende Derma, von hemisphärischer Form, während es beim Lichen planus mehr diffus und ungeordnet ist, sich parallel zur Epidermis erstreekt, so daB die Grenzen zwischen Derma und Infiltrat durch eine gerade Linie dargestellt werden. Die das Infiltrat des Lichen nitidus zusammensetzenden Zellformen findet man auch alle beim Lichen planus (die Lymphozyten und Fibroblasten von epitheloidem Aussehen sind häufig, die Riesenzellen sehr selten). Nie jedoch sind im Infiltrat des Lichen planus die Zellformen derart angeordnet, daß sie die Struktur des Tuberkels reproduzieren, wie es beim Lichen nitidus als Norm vorkommt.

Schlieblich hat A. sklerotısche Alterationen der Blutgefäße beim Lichen nitidus gefunden, die er bei Lichen planus stets vermißte. Alle diese Differenzen sind leicht nachweisbar, wenn die Effloreszenz des Lichen nit. voll entwickelt ist; in früheren Stadien ist die Struktur des Infiltrates wenig charakteristisch und kann verschieden gedeutet werden.

Was die Gefäßalterationen angeht, so tindet man ein Kapillargefä $B$, das zuerst an der Peripherie verläuft und dann in das Innere des Granuloms eintritt, indem es eine stark verdickte Wandung von fast hyalinem Aussehen zeigt. Dieser Befund verdient aus folgenden Gründen beachtet zu werden :

1. Die Gefäßalteration wiederholt sich mit einer gewissen Konstanz (A. konnte sie in fünf Effloreszenzen, die in Serienschnitten untersucht wurden, nachweisen). 
2. Die Alteration trifft nicht alle mit dem Krankheitsherd in Beziehung stehenden Gefäße. Wenn man von den neugebildeten, die peripheren Teile des Infiltrates durchziehenden Kapillaren absieht, so gibt es zum Beispiel vom subpapillären Blutgefäßnetz herkommende und an der Perripherie des Infiltrates verlaufende Gefäße, an denen außer einer mäßigen Schwellung des Endothels keine Alteration nachweisbar ist. Ein einziges Blutgefäß pflegt befallen zu sein und dieses zeigt in den verschiedenen Herden fast die gleiche Topographie.

Die Ätiologie des L. nitidus muß noch anfgeklärt werden. Tuberkelbazillen wurden in den mikroskopischen. Präparaten nie gefunden. Auch Inokulationen von Material des Lichen nitidus hatten negatives Resultat.

Pappagallo, G. Epidermophytia inguinalis (Eczema marginatum Hebra). p. 749.

Nach seinen Untersuchungen in zwölf Fällen kommt A. zu folgenden Schlüssen:

1. Die Epidermophytia inguinalis (Eczema marginatum) ist eine durch pflanzliche Parasiten hervorgerufene, kontagiöse Erkrankung.

2. Sie wird bedingt durch eine besondere Hyphomyzetenart mit bestimmten Eigenschaften, die der Gruppe der Trichophytonpilze nur genähert werden kann. (Die Myzelien, welche in frischen Fällen sehr reichlich rorkommen, haben doppelten Kontur, sind wenig gebogen, fast geradlinig, zeigen dichotomische Verzweigungen und enthalten im Innern viereckige Sporen von verschiedener Größe $-3-5 \mu-$ mit doppeltem Kontur und mehr oder weniger granulösem Protoplasma).

3. Diese Hyphomyzeten nehmen in dér Kultur verschiedenes Aussehen an; es gibt drei Typen:

a) Fidermophyton in zitronengelber,

b) " $\quad$ schmutzig-weißer,

c) " "kastanienfarbener Kultur.

Bosellini, P. L. Tuberculosis cutanea pustulosa follicalaris. p. 758 .

Bosellini gibt in seiner Arbeit einen kasuistischen Beitrag zu den seltenen Formen von suppurierter Hauttuberkulose. Er macht ausdrücklich darauf aufmerksam, daB es sich um eine primäre, durch den Tuberkelbazillus bedingte Suppuration handelte. Ähnliche Fälle sind von Hallopea und 'Thibierge beschrieben worden.

Die Affektion war bei der Patientin des A. am rechten Handrücken und im Gesicht lokalisiert. Es war eine Hauttuberkulose, die unter suppurativ-follikulärer Form aufgetreten war und zwar zuerst mit disseminierten Elementen, die im weiteren Verlaufe durch Hinzutritt neuer Effloreszenzen konfluierten und Flecke bildeten. An der Hand nahm die Dermatose das Aussehen der Tuberculosis verrucosa cutis an, während sie im Gesicht, wo sie anfangs eine spezifische; suppurative Follikulitis bildete, später vegetierende Formen zeigte.

Innerhalb der Läsionen und Pusteln fand sich eine bemerkenswerte Quantität von Tuberkelbazillen (Ha) l opea und Thibierge konnten 
in ihren Fällen den Bazillus nicht nachweisen; die Inokulation des Eiters bei Meerschweinchen hatte jedoch positiven Erfolg).

Da die von A. erhaltene Kultur die Anwesenheit von Staphylokokken in den Pusteln demonstrierte, konnte man sich fragen, welche pathogene Wirkung diesen und welche dem Tuberkelbazillus bei der Bildung der Pusteln zuzuschreiben war. Die experimentelle Impfung brachte keine volle Klarheit. Ein geimpftes Meerschweinchen starb schnell und zeigte pyämische Erscheinungen, die auf die in den kleinen Abszessen auffindbaren Staphylokokken bezogen werden konnten. Bei einem Kaninchen sah man schwere Alterationen der Leber, in denen nebeneinander sehr zahlreiche Kokken und Bazillen vorkamen. Bei zwei Meerschweinchen; die länger lebten, hatte die Tuberkulose das gewöhńliche Aüssehen der experimentellen Tuberkulose.

A. glaubt annehmen zu dürfen, daß der Ko chsche Bazillus das pathogenetische Agens der Pustel war, daß erst sekundär die Staphylokokken, die gewöhnlich bei den feuchten Krankheitsformen zu finden sind, hinzugetreten seien.

Man würde, meint er, auch 'schlecht verstehen, daß reïhliche Lokalisation und Vegetation von Tuberkelbazillen innerhalb einer durch vulgäre Eiterkokken hervorgerufenen Pustel ein sekundäres Faktum sei.

Die beschriebene Form von Folliculitis suppurativa vegetans, die für sich betrachtet, d. h. ohne andere konkomitierende schon bekannte tuberkulöse Läsionen, schwierig zu diagnostizieren ist, weil sie große Ähnlichkeit hat mit einer Folliculitis staphylococcica und in ihrer aggregierten Form mit einer Folliculitis trichophytica, ist auch der von Truffi unter dem Namen Folliculitis staphylogenes vegetans beschriebenen Affektion sehr ähnlich und ruft schließlich die Perifolliculitis agminata suppurativa (Ṕreis), ins Gedächtnis, deren pathologisches Agens ein azidophiler, dem Tuberkelbazillus ähnlicher Bazillus ist. J. Ullmann (Rom).

\section{Polnische Zeitschrift für Haut- und venerische Krankheiten 1910. Band V. Nr. 9.}

Krzysztalowicz, F. Die Plasmazellen (Unna). Nach Angabe der anfänglichen Definition Unnas der Plasmazellen, bespricht K. ihre Genese und Umänderungen in verschiedenen pathologischen Prozessen. Es unterliegt jetzt keinem Zweifel, daß die Plasmazelle ein rein pathologisches Gebilde und für gewöhnlich das Zeichen einer starken progressiven Ernährungsstörung ist. In verschiedenen pathologischen Prozessen begegnet man Bilder, welche die Entstehungsart der Plasmazellen aus den Bindegewebszellen, Endothelien und Perithelien der Blutgefäße illustrieren. Die Vermehrung vollzieht sich durch direkte Teilung, wodurch die sog. Plasmatochterzellen entstehen. Die Plasmazellen können bei 